\title{
Hilfen für den Einstieg in die Pumpentherapie
}

\section{Eine Insulinpumpe kommt derzeit der physiologischen Insulinsekretion am nächsten. Anträge auf Kostenerstattung bei Krankenkassen sind aber nur bei guter Begründung und Dokumentation erfolgreich. Moderne Blutzucker- messsysteme können hier unterstützend wirken.}

Eine Insulinpumpe ist indiziert, wenn der $\mathrm{HbA}_{1 \mathrm{c}}$ trotz intensivierter Insulintherapie (ICT) $>9 \%$ ist, oft schwere, v.a. nächtliche Hypoglykämien oder ein Dawn-Phänomen vorliegen. Auch für schwangere Diabetikerinnen kann eine Pumpentherapie nötig sein.

Basalraten und prandiale Insulingaben können bei der Pumpentherapie völlig voneinander getrennt werden. Moderne Blutzuckermesssysteme wie der AccuChek $^{\bullet}$ Aviva Expert erlauben, verschiedene Basalratenprogramme einzustellen und die Basalrate auch temporär nach Bedarf (z.B. beim Sport) zu verändern. Für die exakte Kalkulation der prandialen Gaben ist ein Bolusrechner integriert.
Der Accu-Chek ${ }^{\circledast}$ Aviva Combo hat die Zusatzfunktionen einer durch Bluetooth gekoppelten Fernbedienung für die Bolusapplikation.

Mit Krankenakten demonstrierte Dr. Dr. med. Wulf Quester, Bad Oeynhausen, dass sich die Glukoseexkursionen nach Umstellung von der ICT auf die Pumpe verringern. Die Patienten haben weniger Hypoglykämien und profitieren von der größeren Flexibilität im Alltag.

Natürlich bedeutet eine Pumpentherapie einen höheren finanziellen Aufwand. Deshalb muss ein relativ aufwendiger Antrag auf Kostenübernahme bei der Krankenkasse gestellt werden, dem als Anlage ein Blutzucker-Tagebuch der letz- ten drei Monate beigefügt werden muss. Die Dokumentation der Daten erleichtert das elektronische Tagebuch im AccuChek $^{\circledast}$ Aviva Expert. Mit der Software Accu-Chek ${ }^{\circledast}$ SmartPix können sie eingelesen und im PDF-Format ausgedruckt werden. Der Arzt kann so prüfen, ob das Tagebuch alle wichtigen Daten enthält, die für die Genehmigung nötig sind.

„Ich habe es kaum je erlebt, dass ein Pumpenantrag, der gut begründet war und eine sorgfältige lückenlose Dokumentation aufwies, abgelehnt wurde“, versicherte Quester. Dr. med. Angelika Bischoff

Seit Juni 2013 stellt Roche Diagnostics ein Aktionspaket zur Verfügung, das die erfolgreiche Beantragung einer Insulinpumpentherapie (CSII) vereinfachen soll. Es enthält eine CSII-Gutachten-Software für Diabetologen, Infobroschüren für $\mathrm{Pa}$ tienten und das Accu-Chek ${ }^{\circledast}$ Aviva Expert. Zudem erhalten teilnehmende Patienten auf Wunsch telefonische Betreuung. Das Paket kann von diabetologischen Praxen mit Pumpenschwerpunkt über den Roche-Außendienst angefordert werden. red

Quelle: Veranstaltung beim Diabeteskongress,

7.5.2013, Leipzig, Veranstalter: Roche Diagnostics u. Pressemeldung Roche Diagnostics v. 5.6.2013
Neue insulinunabhängige Therapie bei Typ-2-Diabetes

Mit der neuen Antidiabetika-Klasse der Gliflozine können Blutzucker, Gewicht und Blutdruck gesenkt werden. Kombiniert mit anderen oralen Antidiabetika oder Insulin ergeben sich additive Wirkungen. Gliflozine sind Hemmer des Transportproteins SGLT-2 in den Nieren. Dadurch wird vermehrt Glukose über den Harn ausgeschieden etwa $70 \mathrm{~g} / \mathrm{d}$, sagte Prof. Andreas Hamann, Bad Homburg. Da Gliflozine wie das bereits in den USA zugelassene Canagliflozin (Invokana ${ }^{\mathrm{TM}}$ ) damit unabhängig von Insulin den Blutzucker senken, ergibt sich in Monotherapie kein relevantes Hypoglykämie-Risiko, so Hamann. Aus bisherigen Studien geht hervor, dass der $\mathrm{HbA}_{1 c}$-Wert im Vergleich zu Placebo im Mittel um ca. einen \%-Punkt gesenkt werden kann, bei schlecht eingestellten Diabetikern gebe es Reduktionen um über 2\%-Punkte. Da mit der Glukose Energie verloren geht, nehmen die Patienten etwa $2-3 \mathrm{~kg}$ ab. Die leicht harntreibende Wirkung senkt den systolischen Blutdruck um bis zu $5 \mathrm{mmHg}$. Hamann zitierte einen Vergleich der Kombinationen Metformin oder Glimepirid mit Canagliflozin. In der 300-mg-Dosis des Flozins ergab sich ein signifikanter Unterschied zur Kontrolle mit einer $\mathrm{HbA}_{1 c}$-Senkung um 0,93 vs. 0,81 \%-Punkte. Auch Dreierkombinationen und jene mit Insulin wurde erfolgreich geprüft. Das EU-Zulassungsverfahren für Canagliflozin läuft. Dr. Thomas Meißner

Quelle: Satellitensymposium beim Diabeteskongress, 8.5.2013 in Leipzig. Veranstalter: Johnson \& Johnson
Gutenberg-Marathon: Diabetesprävention bei Kids im Fokus

Immer mehr Kinder erkranken in Deutschland an Typ-2-

Diabetes. Beim Gutenberg-Marathon, einem sportlichen Großereignis mit hohem Aufklärungswert zur Prävention dieser Volkskrankheit, der von Novo Nordisk unterstützt wird, wurden daher am 12. Mai 2013 die Kinder in den Fokus gerückt.

Vielen Eltern ist nicht bewußt, dass gesunde Ernährung und viel Bewegung das Risiko ihrer Kinder reduzieren, an Typ-2-Diabetes zu erkranken, erklärte Camilla Sylvest, Geschäftsführerin von Novo Nordisk, Mainz. Das Unternehmen engagiert sich seit Jahren mit dem ganzheitlichen

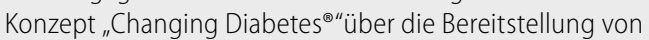
Medikamenten hinaus für die Diabetesprävention. Wie 2012 fand am Tag vor dem Marathon der Mini-Lauf für Kinder statt. Im Rahmen dieser von Novo Nordisk initiierten und vom Ministerium für Bildung, Wissenschaft, Weiterbildung und Kultur des Landes Rheinland-Pfalz unterstützten Aktion besuchte Dr. Harald Schmid, mehrfacher Europa- und Vize-Weltmeister im 400 m Hürdenlauf, mehrere Schulen in Rheinland-Pfalz, die in den vergangenen Jahren erfolgreich am Gutenberg-Marathon teilgenommen haben, spricht mit Schülern über gesunde Ernährung und bietet Ihnen eine Trainingseinheit mit Aufwärmprogramm und Koordinationsübungen an.

Ute Ayazpoor

Quelle: Pressegespräch zum Novo Nordisk Gutenberg-Marathon, 11.4.2013 in Mainz, Veranstalter: Novo Nordisk 